\title{
Experience in Preventive Measures and Treatment of Complications at Face and Neck Thread Rejuvenation
}

\author{
M.A. Sulamanidze, I.S. Vozdvizhenskiy, G.M. Sulamanidze, \\ K.M. Sulamanidze and E.G. Azizyan
}

Additional information is available at the end of the chapter

http://dx.doi.org/10.5772/56250

\section{Introduction}

Face and neck soft tissues lift with the use of special threads is a relatively new and not a thoroughly studied approach to aesthetic surgical and cosmetological correction. Aptos method (anti ptosis) occupies a special place here, because its subjacent conception differs crucially from other thread methods as well as classical surgical ones. Seeming simplicity of intrusion in Aptos method, availability and cheapness of necessary materials and instruments brought a lot of doctors to the point of start in using it, but, unfortunately, without special training. The fact that the doctors do not have special training in Aptos method practice is the main cause of complications and unpleasant aftereffects, the information about which has recently started to appear in literature.

After having studied all the available sources, we have predominantly found the reports about successful results with a minor number of complications and side effects. In the majority of articles both the indications and the intrusion technique are described incorrectly, as well as causes of complications (without considering the anatomical facial special features and kinetics of different groups of muscles), and consequently, this leads to incorrect results.

That is why we have decided to analyze the complications, side-effects and unpleasant aftereffects at face and neck soft tissues thread lift application using Aptos method in various ways. We have analyzed the long-term Aptos method correction experience gained by the surgeons of our clinics (by now there have been performed up to 7000 operations), complications after the application of Aptos among the patients that came from other clinics, and the data presented by doctors from different countries. 
The aim of the conducted work is to diagnose the causes of complications and work out recommendations for the treatment and preventive measures.

\section{Materials and methods}

Out of 900 medical case records taken arbitrarily from the clinical archives of the period from 2002 to 2010 (100 histories per year) only those have been chosen that have data about different complications, problems, and patients' discontent (75 records). According to these documents and the voluminous photo archives of our clinics, a statistical inquiry has been conducted, the results of which are presented in this report along with the commentaries and conclusions. The main products and the corresponding strategies used in our clinics are the following: Aptos thread, Aptos Needle, Aptos Spring, Aptos Thread 2G, Aptos Needle 2G. The manufacturer (Aptos-pro company) usually produces threads from non-absorbable (Prolene) as well as from absorbable (Caprolactone) material. Description of the product, procedure and surgery techniques is given in our numerous publications and presentations $[2-5,10]$, that is why in this article we present short basic information about Aptos methods. This is necessary for understanding the causes of complications, different in nature and severity, and negative manifestations.

Aptos Thread - the first generation of Aptos products - is a thread, $12 \mathrm{~cm}$ long, with convergent angle-wise pins; the thread is fixed subcutaneously with the help of a special hollow needle (figure 1a).

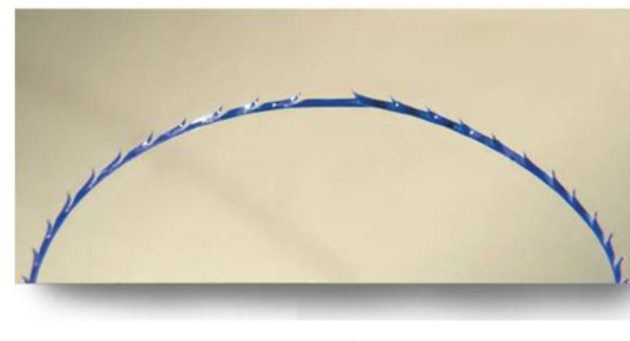

a

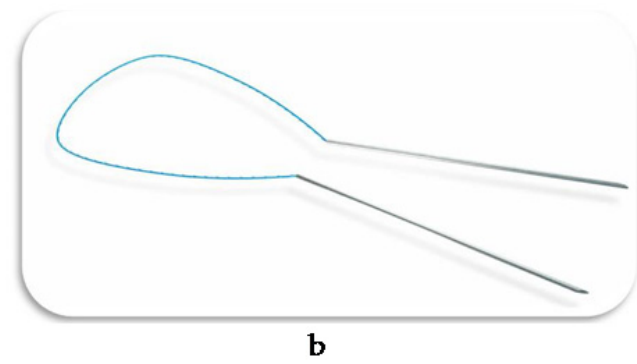

Figure 1. a Aptos Thread, b Aptos Thread 2G

Aptos Thread 2G is the same kind of thread, but $25 \mathrm{~cm}$ long, the ends of which are connected with needles; the needles are sharpened in a special way, their points are joined and put together with temporary glue (figure $1 b$ ). This permits to stick the both needles with the jointed points subcutaneously, separate them at the required depth, and move each needle with the thread along the trajectory, marked beforehand.

Aptos Needle is a unit with a thread, without pins, which is connected with a double-edged needle in its middle part; such a construction permits to put a needle with a thread subcutaneously along the straight, arched or round (tobacco pouch shaped) contour without skin dimpling. The manufacturer produces three types of these needles (figure 2a). 


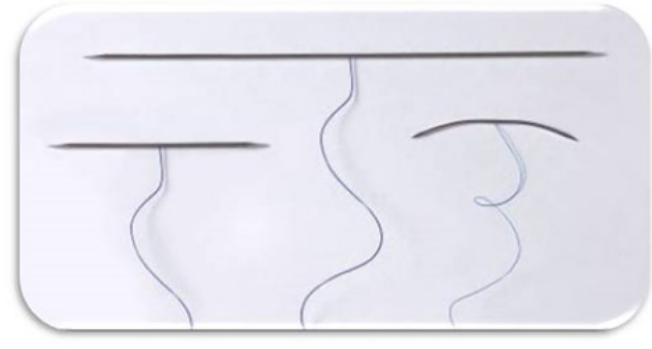

a

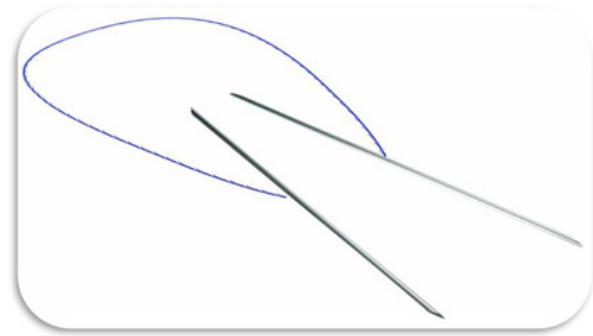

b

Figure 2. a Aptos Needle, Aptos Needle 2G

Aptos Needle 2G combines advantages of Aptos Thread 2G and Aptos Needle. The thread in this case is exactly the same as Aptos thread 2G, but up to $50 \mathrm{~cm}$ long and with convergent pins, connected with two double-edged needles in its middle part (like Aptos Needle), the points of which are put together with temporary glue. That is how the subcutaneous implementation of the thread with pins is performed, and notably, along any contour.

Aptos Spring is a helical elastic thread with "memory" which is used for the lift of the tissues with the most active facial movements.

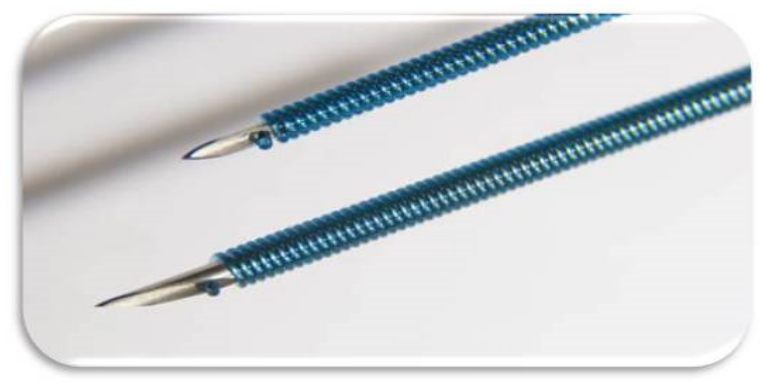

Figure 3. Aptos Springs

\section{Research results and discussion}

Carrying out the research, we have clearly decided on the definitions of complication and inevitable accompanying interference effect. Besides, it was important to understand which complications and disadvantages are characteristics for all aesthetic surgery interferences and which ones are characteristics only for thread lift methods (scheme 1).

For example, we do not regard light paraesthesia, pain, asymmetry and shallow indrawings in the needle puncture places to be complications. Overcorrection is a necessity, a mandatory operation attribute, we have tried to achieve it on purpose in order to stabilize the interference result and make it as long-term as possible (picture $4 \mathrm{a}, \mathrm{b}, \mathrm{c}, \mathrm{d}$ ). 


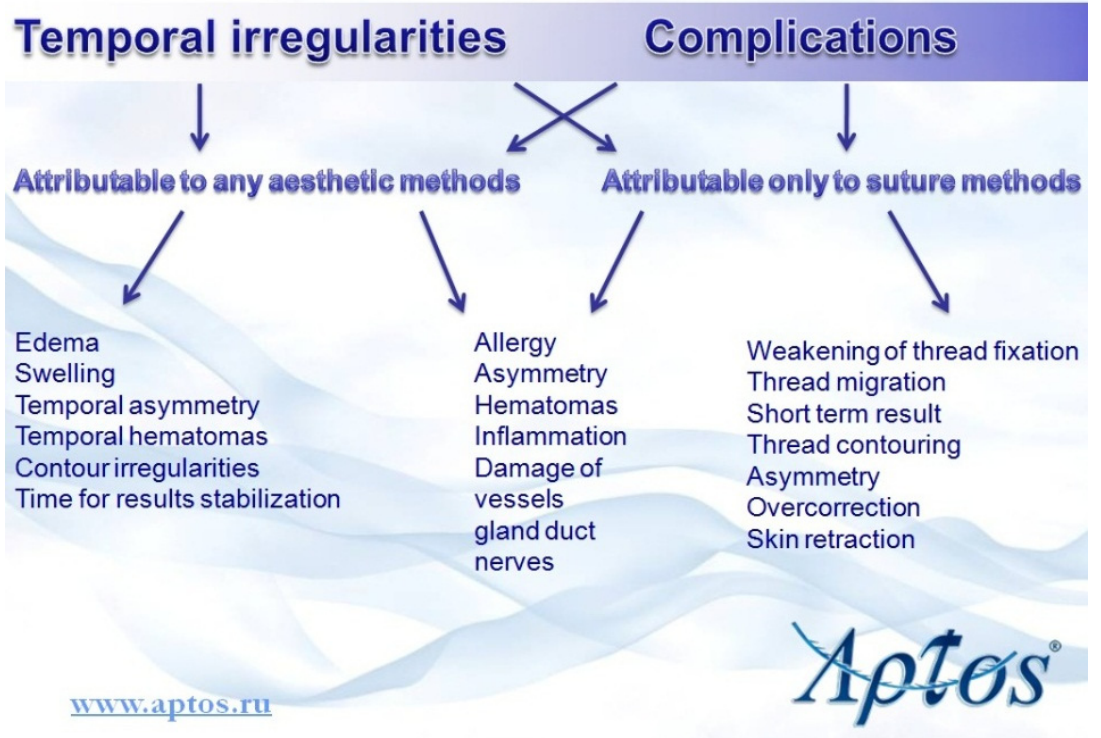

Scheme 1. Classification of Complications and Attendant Thread Lift Negative Factors

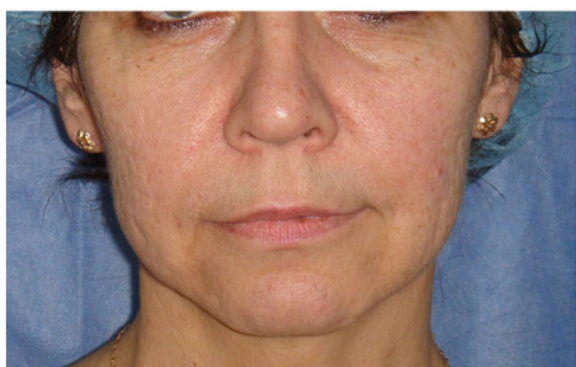

a

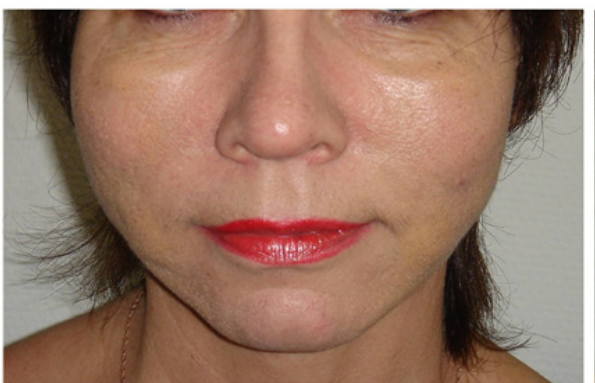

c

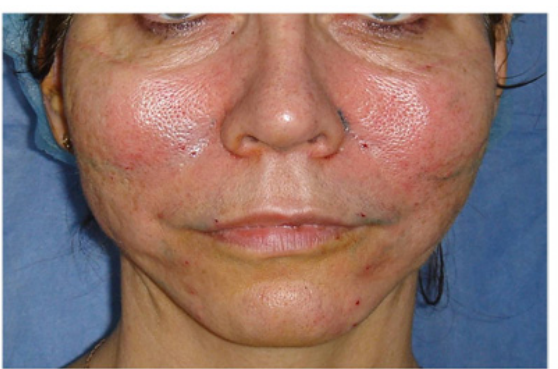

b

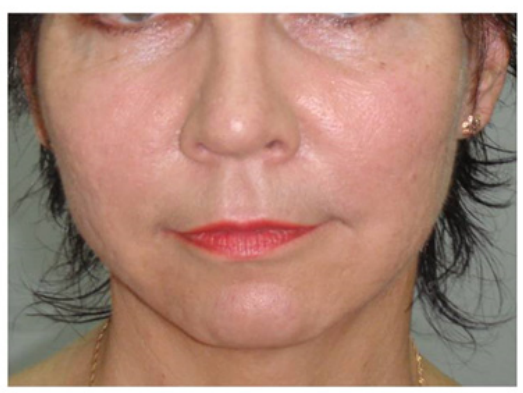

d

Figure 4. Patient N., 38 years old. Middle facial area lift - Aptos Needle 2G, mental area - Aptos Thread 2G, labiomental wrinkles - Aptos Springs: a - before; b - right after the correction; c - in 2 weeks; $d$ - in 2 years after the interference 


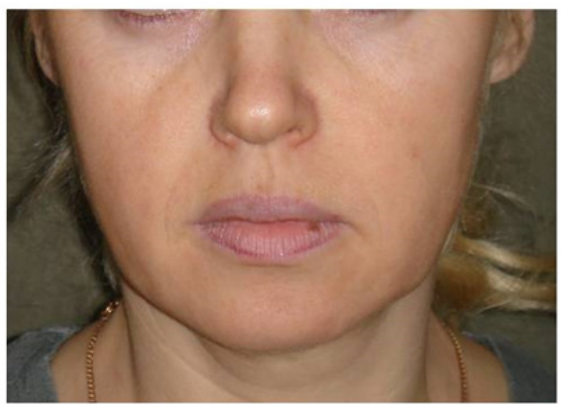

$\mathbf{a}$

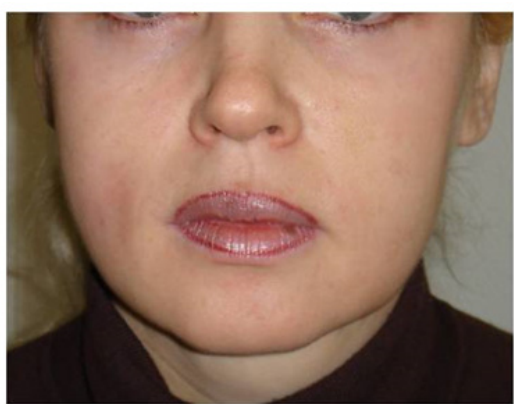

b

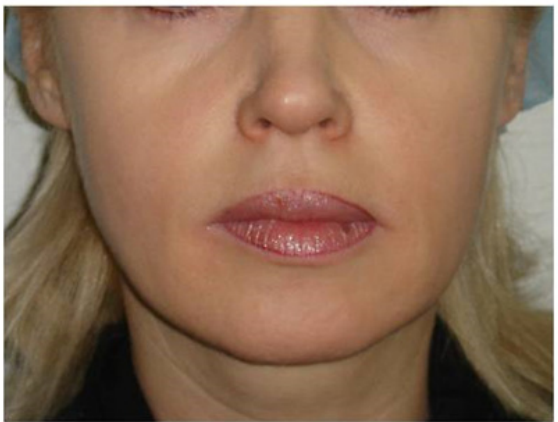

C

Figure 5. Patient A., 44 years old. Middle facial area and mental area lif - Aptos Thread 2G, labiomental wrinkles - Aptos Springs, submandibular and neck areas - Aptos Needle 2/0: a - before, b - in12 days, c - in 3 months after the interference

The interferences can be accompanied by moderate constraint of mimic, masticatory, swallowing and other facial movements, we do not regard these manifestations to be complications either. All the enumerated disadvantages usually go away on its own in dynamics, or you can use common resolving drug therapy or physiotherapy. We present a picture of a patient who had these unpleasant manifestations for 2 weeks, but subsequently the results stabilized and she was satisfied with the performed correction.

We regarded persistent edemata (more than 2-3 weeks), wrong contour, asymmetry, thread visualization, allergy, infection, bleeding along the thread trajectory, thread migration and thread protrusion onto the skin surface, early relapse of soft tissues ptosis, solution of vessels continuity, nerve trunks continuity, and other anatomical structures continuity to be complications.

We threat persistent edemata with additional general clinical methods. Probably, such cases were connected with patients' individual characteristics as it was not always possible to correlate long-term edemata with the severity of undergone surgeries.

In case of wrong contour, indrawn skin areas, if they were not corrected after massage we employed resolving drug therapy and physiotherapy, and eliminated the problem with the 
method of undercutting with the use of a wire scalpel, or corrected it with fillers. The asymmetry was corrected with additional thread lift or, on the contrary, with weakening excessively tight threads (figuree $6 \mathrm{a}, \mathrm{b}$ ). It is most likely that the cause of asymmetry is the surgeon's work fault.

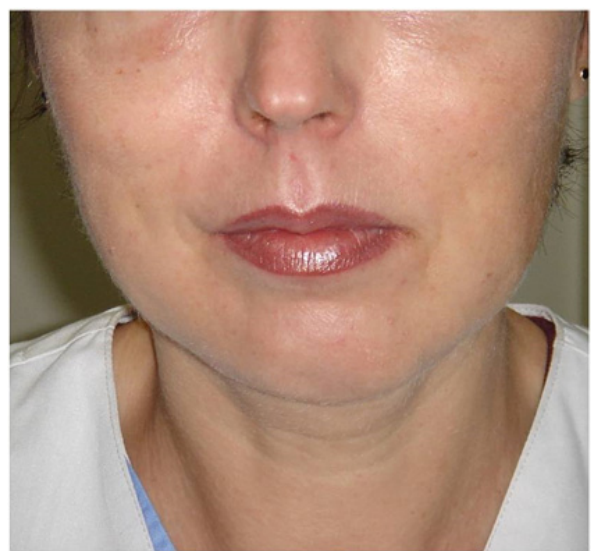

a

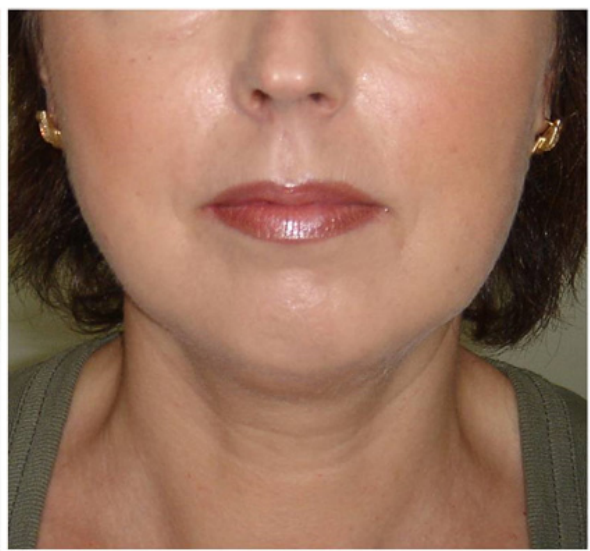

b

Figure 6. Patient L., 47 years oldd. Middle facial area and chin area lift - Aptos Needle 4/0, mental area - Aptos thread 2G, labiomental wrinkles - Aptos Springs.

Two cases of late allergy to the intruded absorbable thread have been marked: the first one is in 10 days, the second one is in 2 months. In the first case the process was terminated with medications, in the second case the threads were removed. (figure 7).
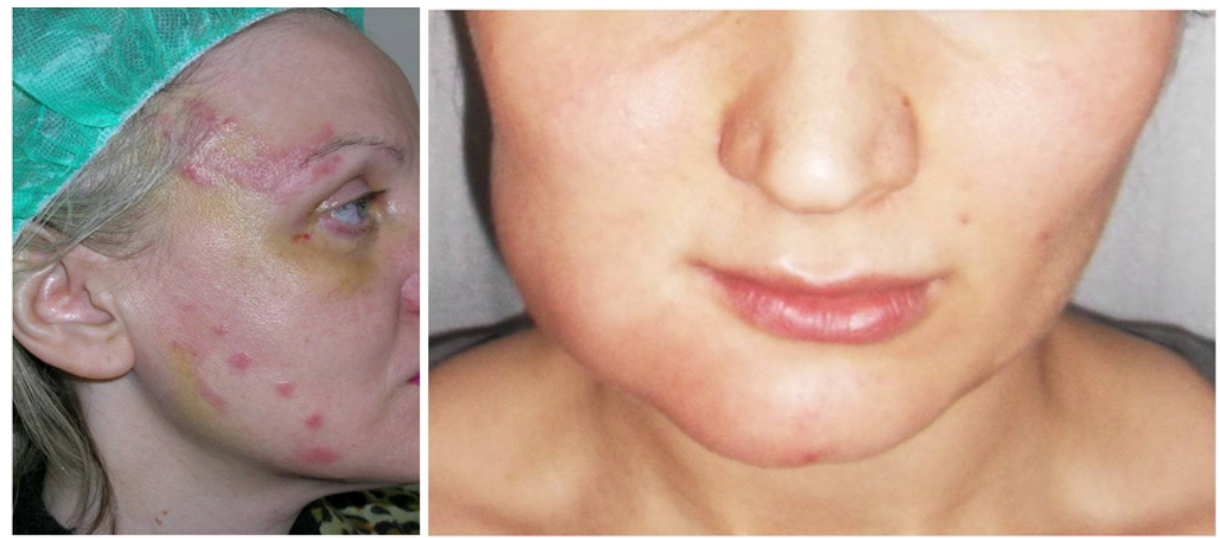

a

b

Figure 7. a Allergic reaction to absorbable threads implementation in 10 days after the interference b. Allergic reaction to absorbable threads implementation in 2 months after the interference 
Migration threads and threads with the end protruded onto the skin surface were also subject to removal (figure 8).

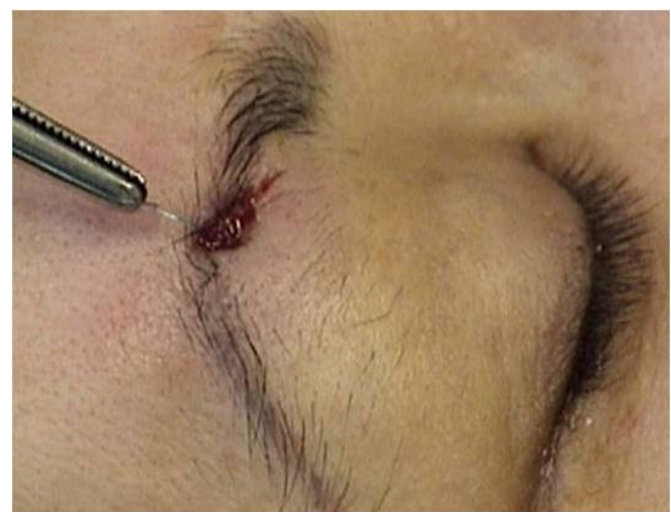

Figure 8. Thread migration case

Since 2008 the usage of prolene Aptos Thread has been stopped and replaced with more modern, second generation threads. And since that time we have not come across any similar unpleasant effects. Such a complication as thread vizualisation has always been connected with its shallow intrusion (except for the eyebrow lifting where this is a necessity). In most cases this problem was successfully eliminated by vigorous massage movements. In rare cases these threads were removed (figure 9).

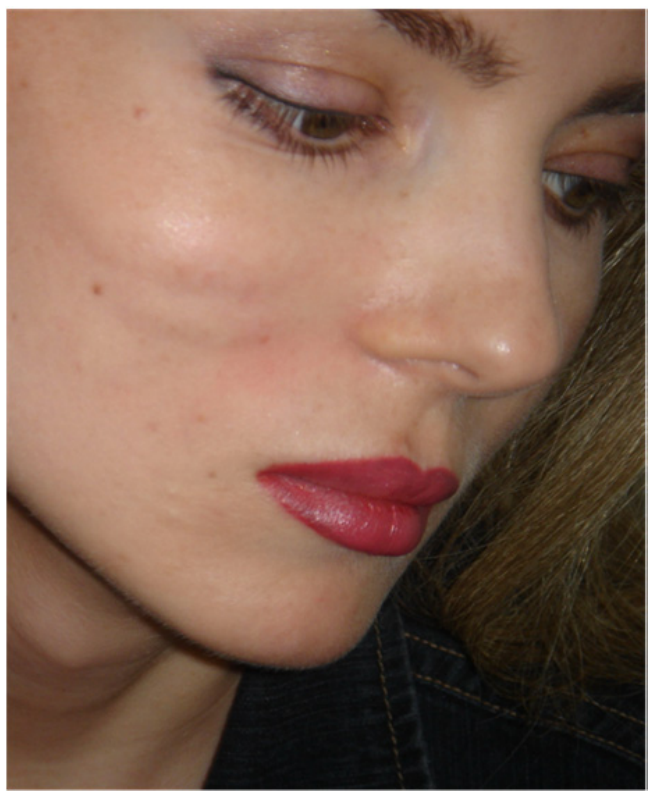

Figure 9. Thread visualization case 
The appearance of complications of inflammatory nature is presumably connected with the infection intrusion into subcutaneous layers during the surgery or post-operative period (figure 10). Preventive measures for such complications involve observation of aseptic and antiseptic rules, following to the doctor's prescription in the post-operative period (taking antibiotics, proper skin care and other recommendations). The treatment is medicamental and general surgical; in case of indication the threads are removed. In our clinic we use noninvasive subcutaneous thread detection methods (US, bright main light visualization, diaphanoscopy) and its mini intrusive removal with the use of a special glover's needle (figure 11). This procedure does not present any technical difficulties for an experienced specialist.

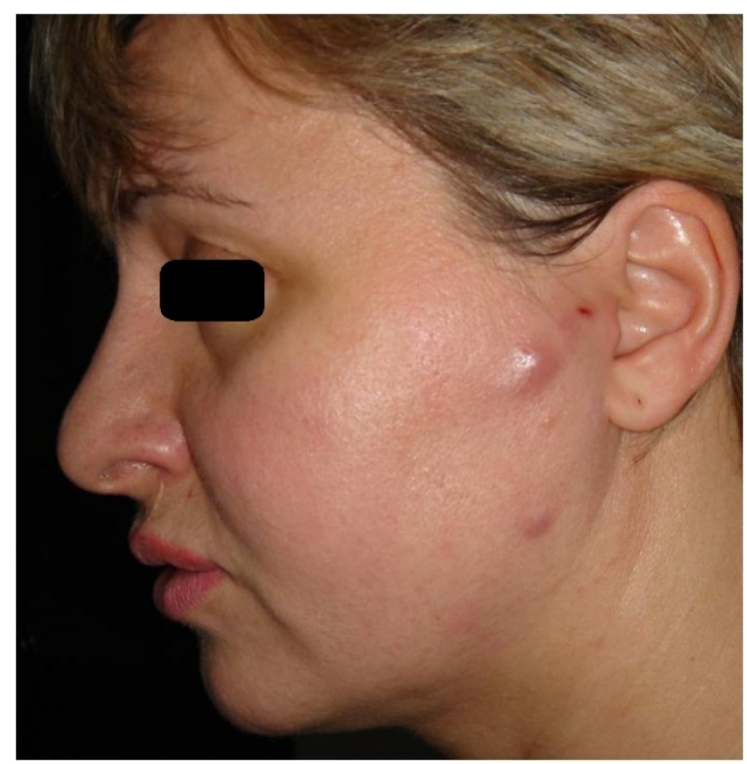

Figure 10. Pus abscess case around thread implementation

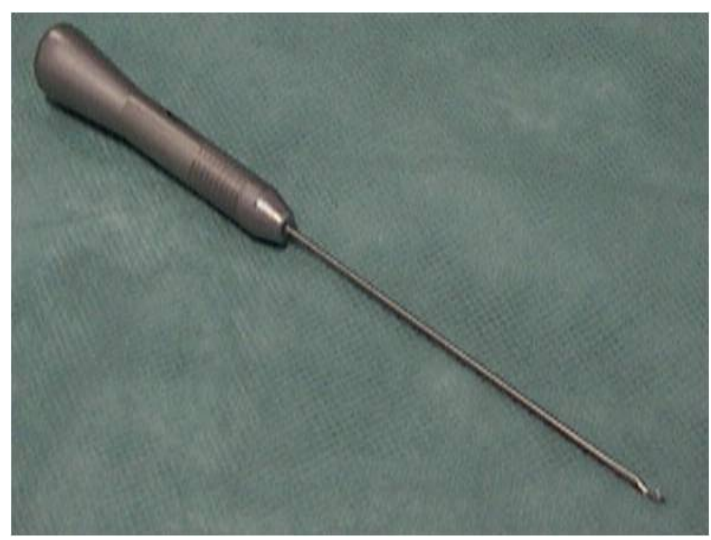

Figure 11. Glover's needle 
In our clinical practice we have not come across the hematomas that needed emptying, macrovessel and nerve trunk continuity solution. However, there is a case of Stenon duct continuity solution described in literature that needed operative surgical intervention in order to be treated [13]. The cases of early soft tissues ptosis relapse are especially worth noticing.

The experience shows that sustainability and stability of thread lift results depend on the observation of a number of rules.

\section{Strict selection of patients according to the indications}

The best face tissues lift results in our clinics are achieved with patients under 50 years old with a slightly thick skin, without pronounced atrophy of subcutaneous fat, with moderate soft tissues ptosis manifestation (feebly marked nasolabial folds and soft-tissue bolsters above them; slightly wrong contour of infraorbital areas in the form of lacrimal grooves; presence of "jowls"), after the preliminary cosmetological deep skin cleansing procedure. For a variety of reasons these patients refused to do classical face lift operations and preferred light and medium face tissues lifts, imperceptible for the others. These were also the patients who were discontent with the previous results of rhytidoplasty, as the face tissues lift was feebly marked in the medial parts and the high volume of bucco-zygomatic areas was not reached.

2. Preoperative Determination of the Interference Goal_(tissue lift, tissue redistribution, creation of a new high volume or combination of these effects)

Aptos methods permit to reach the enumerated goals. For example, eyebrow tail lift and thread canthopexy are performed by Aptos Needle 2G method, while new high volume of the middle part of the face is performed by Aptos Needle 4/0 or Aptos Needle 2G. These methods permit to "border" the lifted tissues area and fix them rigidly to rigid structures.

\section{Fixation of Threads With Pins is Effective Only in the Kinetically Inert Zones}

Eyebrow, bucco-zygomatic, mental, and submandibular areas are among these zones, as they do not have active muscles and soft tissue layers can be easily shifted. That is why rigid lift methods are recommended only in the range of these zones. For the soft tissues lift of the kinetically active zones ("grief wrinkle" correction, mouth corners ptosis) we used an elastic lift - Aptos Springs method.

Simultaneous movement of soft tissue layers of two or more zones is a complicated action, as these zones are separated from each other by dense bands of fibrous tissue that do not allow a long-term lift of all the zones to be performed simultaneously [1,8].

A number of colleagues in their practice ignore these circumstances and employ long threads from temporal or even parietal regions through the whole cheek down to the submandibular area (figure 10a). Of course, during masticatory and mimic movements parts of protuberances or the whole thread are easily destroyed and, consequently, the lift stability declines. Poor results of linear lifts performed by long threads from temporal areas down to the nasolabial triangle and "Marionette Line" can be explained in the same way (figure 10b). 


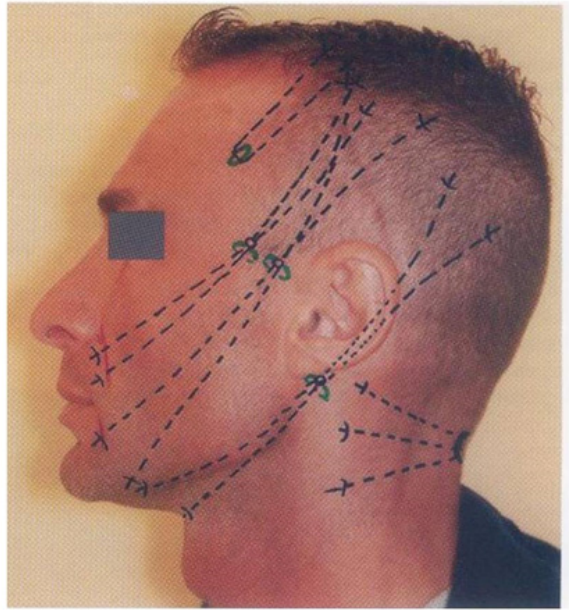

a

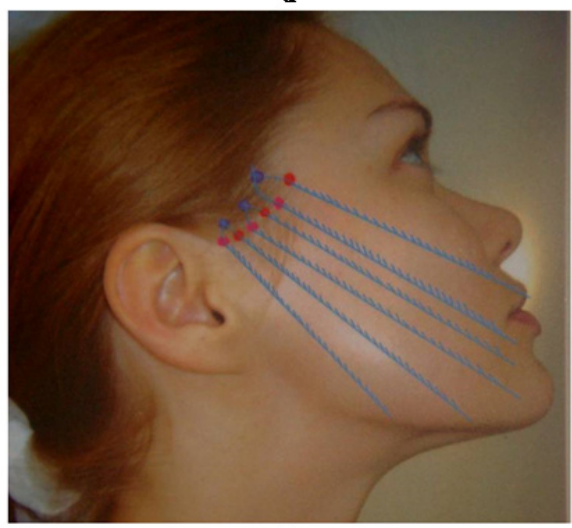

b

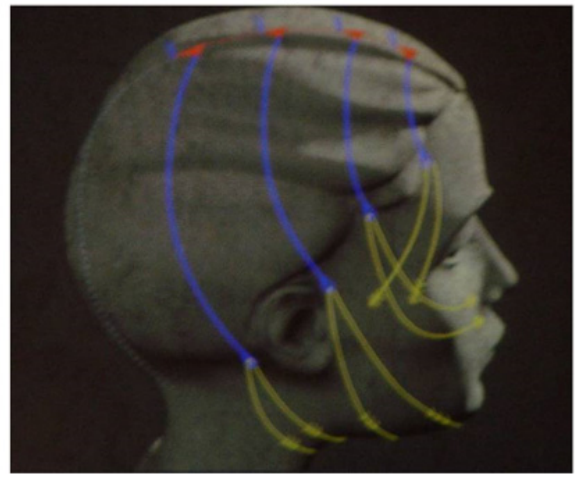

Figure 12. a. Marking before long threads implementatio, $b$. Linear thread direction for the middle facial zone lift, c. Whole face thread lift marking by the method of putting threads over the scalp 
One of the doctors during the performance of the Aptos Thread surgery put long threads from one part of the face over the whole patient's scalp on the other in order to have the threads intruded on the right part of the face could support the threads on the left part of the face and vice versa (figure $10 \mathrm{c}$ ). It is not difficult to imagine the whole operation technology, the process of the practical thread implementation along such a long and difficult contour and the severity of the injury of the tissues along the thread implementation way. Of course, this is not a minimally-invasive intrusion any more [7, 14].

\section{Thread Fixation Depth}

It is determined by the stated objectives, the facial area, the skin depth and the chosen lift thread method. For example, in the case of eyebrow and "jowl" lift threads are fixed rather superficially, while in the bucco--zygomatic and infraorbital areas they are intruded rather deeply into the subcutaneous layer in order to create high volume of the middle facial zone.

Thereby it is possible to confirm that the preventive measures of complications and early relapse cases possible in applying Aptos method are based on the doctor's knowledge of the causes of age and contour facial deformations, the right understanding the idea of Aptos tissue lift, selection of patents according to the indications, the right method choice, irreproachable mastery of equipment, and the delicate intrusion performance. Complication and long-lasting side effects treatment include the achieved results correction (undercutting, implementation of absorbable fillers), elimination of eased and migrating threads, repeated thread or classical lift, physiotherapy, and massage [11, 12].

\section{Conclusions}

The absolute advantages of the Aptos thread method at the lift surgery performance are:

- Simplicity, easiness, efficiency;

- Minimal invasiveness and slight injuring procedures;

- Reliability, sufficient sustainability, and high quality lift;

- Possibility of combination with other interferences;

- Short rehabilitation period.

Complications and unpleasant side effects are not inherent in the very nature of the Aptos method; they are so rare, slight and easily corrected that aesthetic surgery specialists do not have to refuse the usage of lift thread methods.

Some authors present only negative results in their articles about thread lift [6, 9]. However, it must be emphasized that the results of our long-term work absolutely do not agree with the statistics of these publications (Diagrams 2 and 3). The unreasonably suspicious attitude towards the method does not assist the development and improvement of the thread lift methods, and minimally-invasive aesthetic surgery in whole like alternatives to classical lift methods and contour facial surgery. 


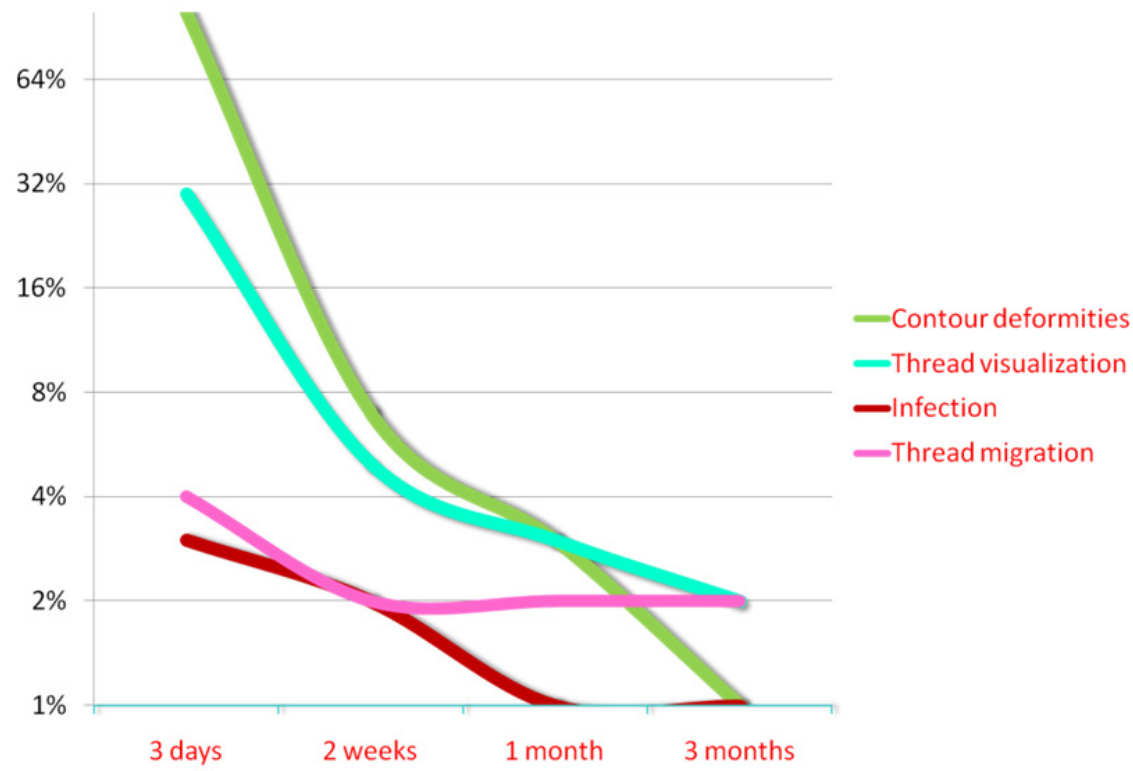

Scheme 2. Frequency and Appearance Dates of Complications in Thread Lift Case

\section{Patient satisfaction of their result.}

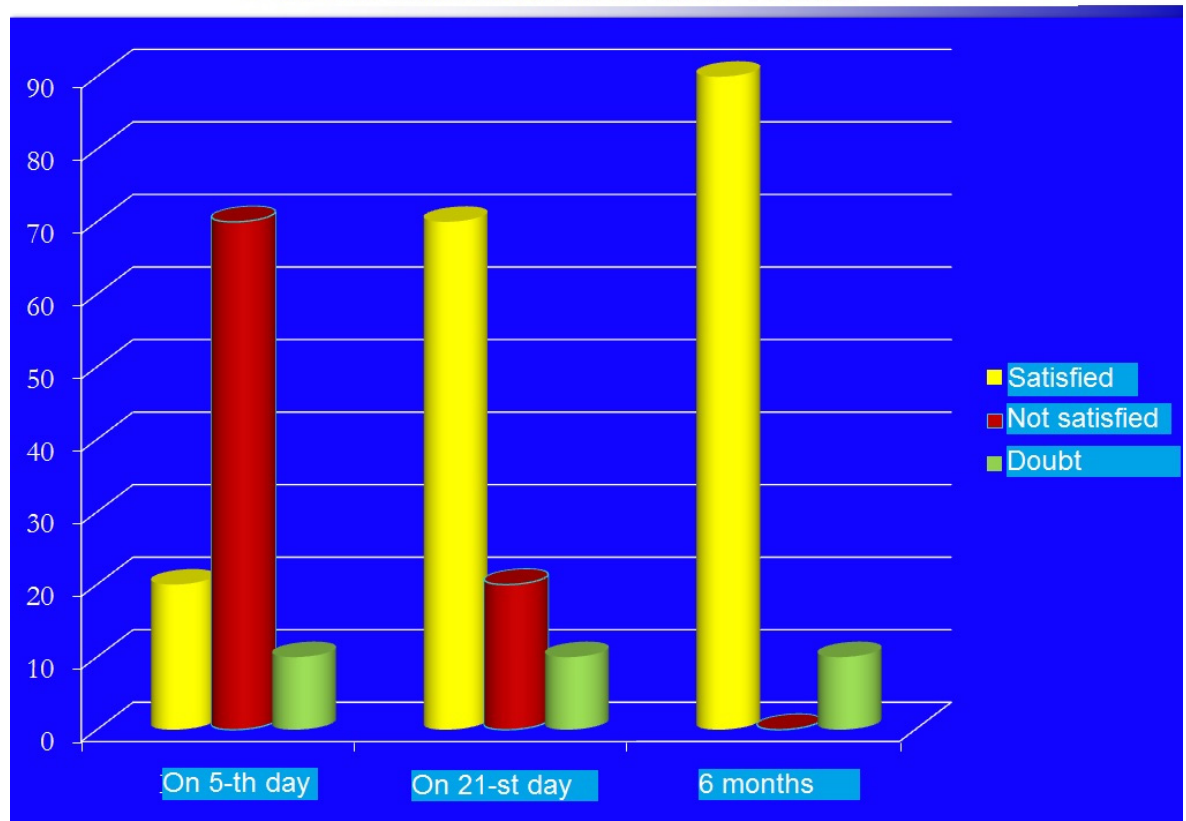

Scheme 3. Patients' Satisfaction with the Results of Thread Lift 


\section{Author details}

M.A. Sulamanidze, I.S. Vozdvizhenskiy, G.M. Sulamanidze,

K.M. Sulamanidze and E.G. Azizyan

Clinic of plastic and Aesthetic Surgery and Dermatocosmetology TotalCharm, Moscow (Russia),

Tbilisi, Georgia

\section{References}

[1] Kazinnikova O.G., Adamian A.A., Age specific changes in facial and cervical tissues // Annals of plastic, reparative, and aesthetic surgery, 2000, 1, 52-61.

[2] Sulamanidze M.A., Sulamanidze G.M. Flabby, ageing face. New approaches // II Congress on aesthetic and restorative surgery, Moscow, 1998:15

[3] Sulamanidze M.A., Sulamanidze G.M. Soft tissues lift: old philosophy, new approach a method of internal stitching (Aptos Needle) // Annals of plastic, reparative, and aesthetic surgery, 2005, 1, 15-29.

[4] Sulamanidze M.A., Sulamanidze G.M. Aptos methods: past, present, future // Aesthetic Medicine Newsletter, 2007, 3, 23-32.

[5] Sulamanidze M.A., Sulamanidze G.M., Vozdvizhenskiy I.S., Sulamanidze K.M., Kadzhaya A.A. New Method of Elastic Thread Face Lift // Aesthetic Medicine, 2010, 3, 275-280.

[6] Aesthetic byers guide. -2006 www.miinews.com

[7] Guillo D. Fils Aptos implantation en X. Surginevs 2005, 8, 10-13.

[8] Mendelson B.C., Muzaffar A.R., Adams W.P. Surgical Anatomy of the Midcheek and Malar Mounds // Plastic. And Reconstruct. Surgery. - 2002, September. - Vol. 110, №3. - P. 885-896.

[9] Rachel, J.D., Lack, E.B., Larson, B. Incidence of complications and early recurrence in 29 patients after facial rejuvenation with barbed suture lifting // Dermatol. Surgery. - 2010. - №36. - P. 348-354.

[10] Sulamanidze M.A., Fournier P.F., Paikidze T.G., Sulamanidze G.M. Removal of Facial Soft Tissue Ptosis With Special Threads// Dermatol. Surgery. - 2002, May. - №28. P.367-371.

[11] Sulamanidze M.A., Sulamanidze G.M. Aptos Suture Lifting Methods. 10 Years of Experience // Clinics in Plast.Surgery. - 2009, April. - Vol.36, №2. - P.281-306.

[12] Sulamanidze M.A., Sulamanidze G.M., Sulamanidze C.M., Vozdvizhensky I.S. Lifting of Soft Tissues With Barbed Threads. Side Effects, Complications. // 11-th ESPRAS Congress Plastic, Restorative and Aesthetic Surgery, 20-26 September 2009, Rhodes, Greece. - Book of Abstracts. - P.25

[13] Winkler E., Goldan O., Regev E. Stenssen duct rupture (sialicele) and other complications of the Aptos thread technique // Plast. Reconstr. Surgery. - 2006. - №118. - P. 1468-71. 
Miniinvasive Face and Body Lifts - Closed Suture Lifts or Barbed Thread Lifts

[14] Woffles Wu. Advances in Stitch Lift // 2-nd Regional Conference in Dermatological Laser and Facial Cosmetic Surgery. - 2006, 20-22 January, Hong Kong. - Programme Book. - P.30 